\title{
SINGULARITIES ON COMPLETE ALGEBRAIC VARIETIES
}

\author{
FEDOR A. BOGOMOLOV, PAOLO CASCINI AND BRUNO DE OLIVEIRA
}

\section{INTRODUCTION}

It is a classical question in algebraic geometry to understand what are the constraints imposed on the singularities that can be afforded on a given class of algebraic varieties. A general result in this direction appeared in $[C G]$. There it was shown that for any algebraic family of algebraic varieties there are isolated singular points which can not be afforded on a any variety which is birationally equivalent to member of this family. Our aim is to prove that any set of isolated algebraic n-dimensional singularities can be afforded on a simply connected projective variety.

More precisely we are going to prove the following result:

Theorem A. Let $(Y, y)$ be the germ of a given isolated singularity. There exists a simply connected projective variety $X$ containing $Y$ and with $X \backslash\{y\}$ smooth.

The variety $X$ we are constructing is of general type and we think that general type condition is necessary in order to afford arbitrary isolated singularity. We will give an interesting example of the result in [CG]. We will describe which sets of rational double points can be afforded on rational surfaces (with the surprising fact that two $E_{6}$ can not be afforded).

This paper is also devoted to what we consider to be a useful description of singularities. We describe the germ of a reduced and irreducible analytic space as a finite cover of a polydisc $\Delta^{n}$ branched along a finite collection of smooth divisors of $\Delta^{n}$ possibly intersecting each other. This picture of singularities provides a good framework to describe the spaces of deformations of isolated singularities and gives a simple proof of the fact that an irreducible and reduced germ of an analytic surface is algebraic.

Another motivation for theorem A was the work of C. Epstein and G. Henkin on the stability of the embeddability property of a strictly pseudoconvex 3-dimensional CR-structure [EH]. More precisely, C Epstein asked the third author if one can always embed an embeddable strictly pseudoconvex 3-dimensional CR-structure inside a regular variety. The methods used

The first author was partially supported by NSF grant DMS-01591 and the third author was partially supported by NSF grant DMS-03693. 
in [EH] see the embeddable CR-manifold $M$ as the boundary of a pseudoconcave surface $Y$ which can be attached to the Stein filling $S$ of $M$ to give a projective variety $X=Y \amalg_{M} S$. The properties of $X$, and specially the regularity, played an important role in their results.

\section{Analytic Singularities}

This section introduces a local description of analytic spaces that we think is very useful to the analysis of a spectrum of problems about singularities. We describe the germ of a reduced and irreducible analytic space as a finite cover of a polydisc $\Delta^{n}$ branched along smooth divisors of $\Delta^{n}$. We give then a new description of the deformation space of an isolated singularity. Another application is a simple proof of the algebraicity of isolated surface singularities.

\subsection{Local Parameterization.}

The following result is a simple modification of the lemma from $[\mathrm{BP}]$ which extends Belyi's argument to the case of arbitrary field of characteristic zero.

Lemma 2.1. Let $Y$ be an $n$-dimensional affine variety. Then there exists a proper map $f: Y \rightarrow \mathbb{C}^{n}$ and a linear projection $p: \mathbb{C}^{n} \rightarrow \mathbb{C}^{n-1}$ such that $f$ is ramified over a finite set of sections $S_{i}$ of $p$.

Proof: Consider an arbitrary finite surjective map $g: Y \rightarrow \mathbb{C}^{n}$. Let $D$ be the ramification divisor of $g$ in $\mathbb{C}^{n}$ and let $p: \mathbb{C}^{n} \rightarrow \mathbb{C}^{n-1}$ be a linear projection whose restriction to $D$ is proper and surjective. The projection $p$ is defined by a point $x \in \mathbb{P}_{\infty}^{n-1}$. To guarantee properness take $x$ outside of the intersection of the closure $\bar{D} \in \mathbb{P}^{n}$ with $\mathbb{P}_{\infty}^{n-1}$. After a linear change of coordinates, $\phi: \mathbb{C}^{n} \rightarrow \mathbb{C}^{n}$, the projection $p$ can be seen as the standard projection onto the last coordinate. Hence, we have a linear parameter $z_{n}$ on all the fibers of $p$ and $p\left(z_{1}, \ldots, z_{n}\right)=\left(z_{1}, \ldots, z_{n-1}\right)$. By Noether normalization, the ramification divisor $D_{0}$ of $g_{0}=\phi \circ g$ is given as the set of zeroes of a monic polynomial $f_{0}\left(z_{n}\right)=z_{n}^{d}+a_{d-1} z_{n}^{d-1}+\ldots+a_{0}=0$ with coefficients $a_{i} \in \mathbb{C}\left[z_{1}, \ldots, z_{n-1}\right], i<n$.

Let $F_{0}: \mathbb{C}^{n} \rightarrow \mathbb{C}^{n}$ be the branch cover of degree d defined by $F_{0}\left(z_{1}, \ldots, z_{n}\right)=$ $\left(z_{1}, \ldots, z_{n-1}, f_{0}\left(z_{n}\right)\right)$ and denote by $g_{1}$ the composition $g_{1}=F_{0} \circ \phi \circ g_{0}$. The ramification divisor of $g_{1}$ is the union of the divisor $z_{n}=0\left(F_{0}\left(D_{0}\right)\right)$ and the divisor $D_{1}=F_{0}\left(R_{0}\right), R_{0}=\left\{\left(f_{0}\right)_{z_{n}}\left(z_{n}\right)=0\right\}$ where $\left(f_{0}\right)_{z_{n}}\left(z_{n}\right)=$ $d z_{n}^{d-1}+(d-1) a_{n-1} z_{n}^{d-2}+\ldots+a_{1}$. The projection $p$ maps the divisor $D_{1}$ properly onto $\mathbb{C}^{n-1}$. The divisor $R_{0}$ has degree $d-1$ with respect to $z_{n}$ and 
hence it's image $F_{0}\left(R_{0}\right)=D_{1}$ is defined by a monic polynomial $f_{1}\left(z_{n}\right)$ of degree $d_{1} \leq(d-1)$ in $z_{n}$.

Let $F_{1}: \mathbb{C}^{n} \rightarrow \mathbb{C}^{n}$ be the branch cover of degree $d_{1}$ defined by $F_{1}\left(z_{1}, \ldots, z_{n}\right)=$ $\left(z_{1}, \ldots, z_{n-1}, f_{1}\left(z_{n}\right)\right)$ and $g_{2}=F_{1} \circ g_{1}$. The ramification divisor is the union of two sections of $p, F_{1}\left(\left\{z_{n}=0\right\}\right)$ and $F_{1}\left(D_{1}\right)=\left\{z_{n}=0\right\}$, and the divisor $D_{2}=F_{1}\left(R_{1}\right)$ which is defined by a monic polynomial on $z_{n}$ of degree $\leq\left(d_{1}-1\right)$. In conclusion, after $i$-step we have the map $g_{i}=F_{i-1} \circ g_{i-1}$ with ramification divisor consisting of the union of $i$ sections of $p$ and a divisor $D_{i}=F_{i-1}\left(R_{i-1}\right)$ which is defined by a monic polynomial on $z_{n}$ of degree $\leq(d-i)$. Therefore, we obtain the lemma after $l \leq d$ steps.

Remark 2.2. The proof of lemma 2.1 also works for a pair $(X, D)$, where $X$ is an arbitrary affine variety of dimension $n$ and $D$ is a divisor of $X$. In this case, the result would be that there is a finite map $f: X \rightarrow \mathbb{C}^{n}$ such that the ramification divisor of $f$ and $f(D)$ are a set sections of a projection $p: \mathbb{C}^{n} \rightarrow \mathbb{C}^{n-1}$.

The previous result can be reformulated in the category of complex analytic spaces to give local results. One such reformulation is a refinement of the Local Parameterization Theorem.

Proposition 2.1 (Local Parameterization). Let $x$ be a point in a complex analytic space $X$ of dimension $n$ and suppose that $X$ is locally irreducible and reduced at $x$. Then $x$ has neighborhood $U \subset X$ with a finite map $f$ : $U \rightarrow \Delta^{n}$ onto an n-polydisc $\Delta^{n}=\Delta^{n-1} \times \Delta$ ramified over a finite collection of sections $S_{i}$ over $\Delta^{n-1}$.

Proof: The standard Local Parameterization Theorem states that all $x \in X$ have a neighborhood $U \subset X$ admitting a finite map $g: U \rightarrow \Delta^{n}$ onto a n-polydisc with $g(x)=(0, \ldots, 0)$. The refinement consists of showing that one can make the ramification divisor of the finite map very well behaved, which provides us with a tool to better understand singularities.

First, we remark that there is nothing to prove if $x$ is not a singular point of $X$. Let $D \subset \Delta^{n}$ be the ramification divisor of the previously described finite map $g: U \rightarrow \Delta^{n}$. We can shrink $U$ and choose a decomposition of the $n$-polydisc $\Delta^{n}=\Delta^{n-1} \times \Delta$ such that the projection of $D$ onto $\Delta^{n-1}$ is a finite mapping. The proof of the standard LP theorem also gives that $D$ is given by a Weierstrass polynomial $f_{0}\left(z_{n}\right)=z_{n}^{d}+a_{d-1} z_{n}^{d-1}+\ldots+a_{0}$ with $a_{i} \in O\left(\Delta^{n-1}\right)$ with $a_{i}=O\left(\left|\left(z_{1}, \ldots, z_{n-1}\right)\right|^{d-i}\right)$.

The previous paragraph provides the setup to apply the method used in the previous lemma. We describe one of the steps to make clear the slight modifications. Using the Weierstrass polynomial $f_{0}\left(z_{n}\right)$ we construct the map $F_{0}: \Delta^{n-1} \times \Delta \rightarrow \Delta^{n-1} \times \Delta^{\prime}$, where $\Delta^{\prime}$ is some disc, given by $F_{0}\left(z_{1}, \ldots, z_{n}\right)=\left(z_{1}, \ldots, z_{n-1}, f_{0}\left(z_{n}\right)\right)$. The map $F_{0} \circ g: U \rightarrow \Delta^{n-1} \times \Delta^{\prime}$ might not be surjective. But by picking a smaller disc $\Delta_{1} \subset \Delta^{\prime}$ and shrinking 
$U$ to $U=\left(F_{0} \circ g\right)^{-1}\left(\Delta^{n-1} \times \Delta_{1}\right)$ we get a finite mapping $g_{1}=F_{0} \circ g$ : $U \rightarrow \Delta^{n-1} \times \Delta_{1}$ ramified at $F_{0}(D)=\left\{z_{n}=0\right\}$ and $D_{1}=F_{0}(R)$ where $R=\left\{\left(f_{0}\right)_{z_{n}}=0\right\}$. The divisor $D_{1}$ is given by the zero set of a Weierstrass polynomial $f_{1}\left(z_{n}\right)=z_{n}^{d_{1}}+a_{d_{1}-1}^{\prime} z_{n}^{d_{1}-1}+\ldots+a_{0}^{\prime}$ of degree $d_{1} \leq d-1$. Use $f_{1}\left(z_{n}\right)$ to construct $F_{1}$ and do the necessary shrinking of $U$, as before, and obtain a finite map $g_{2}=F_{1} \circ g_{1}: U \rightarrow \Delta^{n-1} \times \Delta_{2}$. The desired finite map $f$ will be the map $g_{l}: U \rightarrow \Delta^{n-1} \times \Delta_{l}$ obtained after some $l \leq d$ steps.

\subsection{Applications.}

In this section we show how to apply proposition 2.1 to obtain the algebraicity of the germs of normal 2-dimensional complex spaces and give a description of isolated singularities that might prove to be useful for the description of their deformations.

The Local Parameterization theorem presented in section 2.1 provides a simple proof of the algebraicity of any germ of an analytic surface. For isolated singularities this is a well known result due to Artin [A2] and later extended to a global result by Lempert [Le]. More precisely, Lempert showed that any reduced Stein space $S$ with boundary $\partial S=M$ a smooth CRmanifold can be embedded in an algebraic variety. On the other hand, recall that in [W] (examples 14.1 and 14.2) Whitney shows that analytic singularities in dimensions $n \geq 3$ are, in general, not locally algebraic. Whitney constructs an example of a normal analytic variety $V$ of dimension 3 and with a singular point $p \in V$, such that there exists no algebraic variety that is locally (in an analytic sense) biholomorphic to any open neighboorhood of $p$ in $V$.

We proceed to show that all the analytic singularities in dimension 2 are locally algebraic. Let $p$ be a point of a complex analytic surface $S$, and suppose that $S$ is normal at $p$. By Proposition 2.1, there exists an open neighborhood $U \subseteq S$ of $p$, admitting a finite map $g: U \rightarrow \Delta^{2}$, where $\Delta^{2}$ is a polydisc in $\mathbb{C}^{2}$ and such that $g(p)=(0,0)$.

If the ramification divisor of $g, D \subset \Delta^{2}$, was an algebraic curve on $\Delta^{2}$ (i.e. given by the zero locus of a polynomial), then $U$ would be an open subset of an algebraic surface. But $D$ is possibly reducible an analytic curve in $\Delta^{2}$. To deal with this case, we have:

Lemma 2.3. Let $D \subseteq \Delta^{2}$ be a reduced analytic divisor, such that $(0,0) \in D$. Then, up to shrinking $\Delta^{2}$, there exists a biholomorphic map from $\Delta^{2}$ onto an open neighborhood $V$ of $(0,0)$ in $\mathbb{C}^{2}$ such that the image $D^{\prime}$ of $D$ is an algebraic divisor passing through $(0,0)$. 
Proof: Levinson proves a more general result in [L] (see also [W], remark 14.3). But, for the sake of completeness, we show an easy proof of the lemma. Let $D$ be a union of irreducible components $D_{i}$, with $i=1, \ldots, N$, passing through $(0,0)$.

By choosing a suitable system of coordinates $z_{1}, z_{2}$ and after shrinking the polydisc $\Delta^{2}$, we can suppose that if $p_{1}: \Delta^{2} \rightarrow \Delta$ is the projection with respect to the first coordinate, then each $D_{i}$ is a section of $p_{1}$. In other words, we can write each $D_{i}$ as the zero set of the function $F_{i}\left(z_{1}, z_{2}\right)=z_{2}-f_{i}\left(z_{1}\right)$, with $f_{i}$ analytic.

We want to prove the lemma by induction on $N$ (the number of irreduible components of $D)$. Suppose that $f_{1}, \ldots, f_{k}$ are polynomials, with $k<N$. We want to construct a biholomorphism of the form

$$
\Phi\left(z_{1}, z_{2}\right)=\left(z_{1}, z_{2}+g\left(z_{1}, z_{2}\right) \prod_{i=1}^{k} F_{i}\left(z_{1}, z_{2}\right)\right)
$$

where $g$ is an analytic function such that $\Phi\left(D_{k+1}\right)$ is algebraic. In fact, by construction it follows that $\Phi\left(D_{i}\right)=D_{i}$ for any $i \leq k$.

In order to reach our aim, we have to choose $g$ such that the analytic function

$$
P\left(z_{1}\right)=f_{k+1}\left(z_{1}\right)+g\left(z_{1}, f_{k+1}\left(z_{1}\right)\right) \prod_{i=1}^{k}\left(f_{k+1}\left(z_{1}\right)-f_{i}\left(z_{1}\right)\right)
$$

is indeed a polynomial.

By shrinking $\Delta^{2}$ again, if necessary, we can suppose that

$$
\prod_{i=1}^{k}\left(f_{k+1}\left(z_{1}\right)-f_{i}\left(z_{1}\right)\right)=z_{1}^{M} \phi\left(z_{1}\right)
$$

for some $M>0$ and $\phi$ analytic function such that $\phi(0) \neq 0$.

Therefore we can find an holomorphic function $g$, satisfying (1), for any polynomial $P$ such that $P\left(z_{1}\right)-f_{k+1}\left(z_{1}\right)$ is divisible by $z_{1}^{M}$.

Our claim follows from the lemma. Choose $U^{\prime}$ defined by $h^{-1}\left(\Delta^{2}\right)$, where $h=\Phi \circ g: U \rightarrow V$ and $(0,0) \in \Delta^{2} \subset V$, as the neighborhood of $p$. The open set $U^{\prime}$ is a branched covering of $\Delta^{2}$ branched over an algebraic curve.

The Local Parameterization result described in proposition 2.1 gives directly the following description of isolated singularities.

Proposition 2.2. Let $s$ be a normal isolated singularity in a n-dimensional complex analytic space $Y$. Then: 
a) There is an open neighborhood of $s, U \subset Y$, admitting a finite map $f: U \rightarrow \Delta^{n}$, onto an n-polydisc, which is unramified outside of a finite set of smooth subvarieties $S_{i} \subset \Delta^{n}$.

b) The germ of the singularity $s \in Y$ is determined by the pair $\left(\Delta^{n}-\right.$ $\left.\bigcup_{i}^{k} S_{i}, \Gamma\right)$, where $\Gamma$ is the subgroup of finite index of $\pi_{1}\left(\Delta^{n}-\bigcup_{i}^{k} S_{i}\right)$ defining the covering.

The above picture of a singularity can be quite useful to determine the structure of the deformation space for many isolated singularities. Let $(Y, s)$ be the germ of a normal n-dimensional singularity corresponding to the pair $\left(\Delta^{n} \backslash \bigcup_{i}^{k} S_{i}, \Gamma\right)$. Denote by $s_{i} \in \pi_{1}\left(\Delta^{n} \backslash \bigcup_{i}^{k} S_{i}\right)$ the simple loops around the irreducible components $S_{i}$. The $s_{1}, \ldots, s_{k}$ generate $\pi_{1}\left(\Delta^{n} \backslash \bigcup_{i}^{k} S_{i}\right) \cong$ $\mathbb{Z}^{m}$, where $m$ is the multiplicity of the irreducible holomorphic function germ $g$ with $g^{-1}(0)=\bigcup_{i}^{k} S_{i}$. Let $\Gamma^{\prime}$ be the maximal normal subgroup of $\pi_{1}\left(\Delta^{n} \backslash \bigcup_{i}^{k} S_{i}\right)$ contained in $\Gamma$. The next short exact sequence holds:

$$
1 \rightarrow \Gamma^{\prime} \rightarrow \pi_{1}\left(\Delta^{n} \backslash \bigcup_{i}^{k} S_{i}\right) \rightarrow G \rightarrow 1
$$

where $G$ is the Galois group of the cover induced by $\Gamma^{\prime}$.

Consider a deformation $\bigcup_{i}^{k} S_{i}^{t}$ of $\bigcup_{i}^{k} S_{i}$. Let $T$ be a tubular neighborhood of $\bigcup_{i}^{k} S_{i}$. The complement $\Delta^{n} \backslash T$ is homotopically equivalent to $\Delta^{n} \backslash \bigcup_{i}^{k} S_{i}$ and it is immersed in $\Delta^{n} \backslash \bigcup_{i}^{k} S_{i}^{t}$ for $\frac{1}{|t|} \gg 0$. Hence there is a natural homomorphism $j_{t}: \pi_{1}\left(\Delta^{n} \backslash \bigcup_{i}^{k} S_{i}\right) \rightarrow \pi_{1}\left(\Delta^{n} \backslash \bigcup_{i}^{k} S_{i}^{t}\right)$ for $\frac{1}{|t|} \gg 0$.

Assume that a surjection $r_{t}: \pi_{1}\left(\Delta^{n} \backslash \bigcup_{i}^{k} S_{i}^{t}\right) \rightarrow G$ holds and moreover that $r_{t} o j_{t}: \pi_{1}\left(\Delta^{n} \backslash \bigcup_{i}^{k} S_{i}\right) \rightarrow G$ is constant for $\frac{1}{|t|} \gg 0$. This implies that a Galois cover, associated with $G$, of $\Delta^{n} \backslash \bigcup_{i}^{k} S_{i}^{t}$ persists for small $t$ and the induced covering of $\Delta^{n} \backslash T \subset \Delta^{n} \backslash \bigcup_{i}^{k} S_{i}^{t}$ is constant along the family. In turn, this implies that an intermediate covering associated with $\Gamma$ inducing a constant covering of $\Delta^{n} \backslash T \subset \Delta^{n} \backslash \bigcup_{i}^{k} S_{i}^{t}$ also persists for small $t$.

The end result is that from a family of divisors $\bigcup_{i}^{k} S_{i}^{t} \subset \Delta^{n}$ for which $r_{t} o j_{t}: \pi_{1}\left(\Delta^{n} \backslash \bigcup_{i}^{k} S_{i}\right) \rightarrow G$ is constant for $\frac{1}{|t|} \gg 0$ one obtains a family of singularities $Y_{t}$ associated with the pairs $\left(\Delta^{n} \backslash \bigcup_{i}^{k} S_{i}^{t}, \Gamma\right)$. The singularities $Y_{t}$ all have a finite map $f_{t}: Y_{t} \rightarrow \Delta^{n}$ of the same degree branched at $\bigcup_{i}^{k} S_{i}^{t}$. Moreover, the $Y_{t}$ have an arbitrarily large open subset $f_{t}^{-1}\left(\Delta^{n} \backslash T\right) \subset$ $f_{t}^{-1}\left(\Delta^{n} \backslash \bigcup_{i}^{k} S_{i}^{t}\right)$ which is biholomorphic to $f_{0}^{-1}\left(\Delta^{n} \backslash T\right)$ for all sufficiently small $t$. The conditions to impose on the $S_{i}^{t}$ to guarantee the constancy of $r_{t} o j_{t}: \pi_{1}\left(\Delta^{n} \backslash \bigcup_{i}^{k} S_{i}\right) \rightarrow G$ will be investigated in future work. 


\section{Singularities inside Projective varieties}

Any collection of isolated singularities can be afforded in some projective variety (see paragraph below). On the other hand, a collection of singularities, or even one single singularity, does impose global constraints on the type of the variety that possesses it (see the next subsection). The main goal of this section, theorem A, is to show that the property of being simply connected is not one of the properties which is conditioned by the presence of singularities. Along the same lines would like to conjecture a stronger result:

Conjecture 3.1. Let $(Y, y)$ be the germ of a given isolated singularity. There exists a projective variety $X$ containing $Y$ and with $X \backslash\{y\}$ smooth whose resolution $\hat{X}$ is simply connected.

The following lemma shows that every finite set of isolated singularities, can be afforded in an unique projective variety.

Lemma 3.1. Let $\Gamma=\left\{\left(Y_{i}, y_{i}\right)\right\}_{i=1, \ldots, k}$ be any collection of germs of algebraic isolated singularities of dimension $n$. There exists a projective variety $Y$ having $\Gamma$ as its singular locus.

Proof Let $X_{k}$ be a variety with only one singular point and the germ of the singularity is equivalent to $\left(Y_{k}, p_{k}\right)$. The lemma follows from induction. Assume we constructed a projective variety $Y_{k-1}^{\prime}$ with $\Gamma_{k-1}=$ $\left\{\left(Y_{i}, y_{i}\right)\right\}_{i=1 \ldots k-1}$ as its singular locus. Consider the product variety $Y_{k-1}^{\prime} \times$ $X_{k}$ and cut it by a general $n$-codimensional plane $H_{n}$ and denote $Y_{k}^{\prime}=$ $Y_{k-1}^{\prime} \times X_{k} \cap H_{n}$. The singularities of $Y_{k}^{\prime}$ are isomorphic to the $\left(Y_{i}, y_{i}\right)_{i=1 \ldots k}$ are by a dimensional and general position argument. $H_{n}$ intersects transversely the singular locus $p_{i} \times X_{k}$ or $Y_{k-1}^{\prime} \times p_{k}$ and avoids the points $p_{i} \times p_{k}$. If we are interested in having exactly one copy of each singularity then resolve the possible extra copies of the singularities $\left(Y_{i}, y_{i}\right)_{i=1 \ldots k}$ that might occur.

\subsection{An example of constraints imposed by singularities.}

In the introduction we recalled a recent result of Ciliberto and Greco stating that for any algebraic family of algebraic varieties there are isolated singular points which can not be afforded on a any variety birational to a member of this family. We proceed to give a concrete example of this result. More precisely, we describe all the sets of rational double points, RDP's, 
that a rational surface can contain (the same result holds for all surfaces of Kodaira dimension $-\infty)$.

Notation: Let $X$ and $Y$ be analytic normal complex surfaces and $f$ : $Y \rightarrow X$ be a birational morphism with exceptional set $E=\sum E_{i}$. The negative definiteness of the intersection matrix $\left(E_{i}, E_{j}\right)$ allow the existence of a unique solution to:

$$
K_{Y} \equiv f^{*} K_{X}+\sum a_{i} E_{i}
$$

The numbers $a_{i}$ are called the discrepancy of $E_{i}$ with respect to $X$, $\operatorname{discrep}\left(E_{i}, X\right)=a_{i}$. The birational morphism will be called totally discrepant if $E \neq \emptyset$ and the $a_{i}>0$, for all $i$.

It was shown is Sakai [S1] that given a normal surface $X$, there is a sequence $X \rightarrow X_{1} \rightarrow X_{2} \rightarrow \ldots \rightarrow X_{n}$ of contractions of exceptional curves of the first kind, i.e $C^{2}<0$ and $K_{X_{i}}$. $C<0$, such that $X_{n}$ has no such curves. $X_{n}$ is then called a minimal model of $X$ and the morphism $f: X \rightarrow X_{n}$ is totally discrepant. Let $X^{\prime}$ be the minimal model of the normal surface $X$ and $f: X \rightarrow X^{\prime}$ be the totally discreptant birational morphism. Let $\pi^{\prime}: Y^{\prime} \rightarrow X^{\prime}$ and $\pi: Y \rightarrow X$ be respectively the minimal resolutions of $X^{\prime}$ and $X$, with $K_{Y^{\prime}}=\pi^{\prime *} K_{X^{\prime}}+\Delta^{\prime}$ and $K_{Y}=\pi^{*} K_{X}+\Delta$. Then $f$ induces a birational morphism $g: Y^{\prime} \rightarrow Y$ such that $g_{*} \Delta \geq \Delta^{\prime}$ (this result supports the statement that going to the minimal model does not make singularities worse).

A normal surface singularity $\left(X, x_{0}\right)$ is an RDP (rational double point) iff $K_{Y} \cdot E_{i}=0$ for every exceptional curve $E_{i}$ of the minimal resolution $f: Y \rightarrow X$ or equivalently $f^{*} K_{X}=K_{Y}+\Delta$ with $\Delta=0$. From the definition of an RDP singularity follows that the negative configuration of curves that form the exceptional set of $f$ is composed of smooth rational curves with self intersection -2 in one of formations of the Dinkin diagrams $A_{n}(n=1, \ldots), D_{n}(n=4, \ldots), E_{n}(n=6,7,8)$. The observation of the previous paragraph implies that if a normal surface $X$ has only RDP singularities then the same is true for its minimal model.

Theorem B. The collection of rational double points that can be in a rational surface $X$ are the following:

1. Arbitrary collections of $A_{n}$ and $D_{n}$ singularities

2. An $E_{n}$ singularity and an arbitrary collections of $A_{n}$ singularities.

Proof First, we give the positive results. By blowing up over a point one can get an $A_{n}$ configuration of negative curves. Hence all birational classes of surfaces can have as many $A_{n}$ singularities as desired. 
A $D_{n}$ configuration of negative curves can be obtained by blowing up over a smooth rational curve $C$ with $C^{2}=0$. Hence one can get as many $D_{n}$ singularities as desired in all birational classes of ruled surfaces.

An $E_{n}$ configuration can not be obtained by blowing up over a smooth rational curve $C$ with $C^{2}=0$. This is the reason behind the asymmetry of the theorem. On the other hand, one $E_{n}$ configuration of negative curves can be obtained by blowing up over two lines in $\mathbf{P}^{2}$. Hence one can get one $E_{n}$ singularity in the birational class of rational surfaces.

The minimal model program for normal singular surfaces developed by Sakai will give the negative results. Let $Y$ be a normal surface with two $E_{n}$ singularities or an $E_{n}$ and one $D_{m}$ singularity. Resolve all the other singularities and still name that surface $Y$. The minimal model $Y_{m}$ of $Y$ is a surface with only rational double points, as explained above. Moreover, the singularities $E_{n}$ and $D_{m}$ of $Y$ will still exist in the minimal model $Y_{m}$ [Mo]. Let $f: X \rightarrow Y_{m}$ be the minimal resolution of $Y_{m}$ (i.e. no (-1)-curves on the exceptional locus). Assume, as in the hypothesis of the theorem, that $X$ is rational, then since the singularities of $Y_{m}$ are rational, $K_{X}=f^{*} K_{Y_{m}}$, one has $\operatorname{Kod}\left(Y_{m}\right)=-\infty$ and $K_{Y_{m}}$ is not nef.

Sakai [S2] proved that if $Y^{\prime}$ is a minimal normal surface whose canonical bundle $K_{Y^{\prime}}$ is not nef then $Y^{\prime}$ is projective, $\operatorname{Kod}\left(Y^{\prime}\right)=-\infty$ and either:

i) $\rho(W)=1$ and $-K_{W}$ is numerically ample, i.e. $K_{W}^{2}>0$ and $K_{W} \cdot C>0$ for all curves $C \subset W$, or

ii) $W$ has a $\mathbb{P}^{1}$-fibration.

So according to Sakai's result $Y_{m}$ must be one of the two cases described above. We will show that both cases are not possible.

Suppose $Y_{m}$ is as in i). The minimal resolution $X$ of $Y_{m}$ is rational and has $K_{X}^{2}=\left(f^{*} K_{Y_{m}}\right)^{2}>0$ Hence $X$ is $\mathbb{P}^{2}$ blown up at most 8 times or one of the Hirzebruch surfaces $F_{n}$ blown up at most 7 points. In both cases $b_{2}^{-}(X) \leq 8$. But on the other hand the minimal resolution of $Y_{m}$ must have $b_{2}^{-}(X) \geq n+m \geq 10$ and we obtain a contradiction. The inequality is just a consequence of the linear independence of homology classes of the curves in the exceptional locus.

Suppose $Y_{m}$ is as in 2). The $\mathbb{P}^{1}$-fibration of $Y_{m}$ induces a ruled-fibration, $\pi: X \rightarrow C$, of $X$. The configuration of (-2)-curves coming from the resolution of the $E_{n}$ singularity lies in one of the fibers. The surface resulting from contracting the (-1)-curves in the fibers of $\pi$ is an Hirzebruch surface $F_{n}$. But an $E_{n}$ configuration of (-2)-curves can not be obtained by blowing up over a smooth rational curve $C$ with $C^{2}=0$ and the desired contradiction follows. 
Corollary 3.1. A surface $X$ which is a resolution of a surface $Y$ containing $a E_{n}$ and $a D_{n}$ singularity must have its Kodaira dimension $\operatorname{Kod}(X) \geq 0$.

Proof: The last theorem states that $X$ is not a rational surface. On the other hand, an $E_{n}$ configuration of negative curves does not lie entirely in the blow up pre-image of a fiber of a ruled surface. This would force one of the $(-2)$-curves to surject to the base of the ruled surface imposing that $X$ is rational.

Corollary 3.2. There is a singularity that can not be afforded in a projective surface $X$ with $\operatorname{Kod}(X)=-\infty$.

Proof: Let $X^{\prime}$ be a smooth projective surface with a $E_{n}$ and a $D_{m}$ configurations of -2-curves which are disjoint. Let $H$ be an ample divisor on $X^{\prime}$, blow up $X^{\prime}$ at a sufficiently large number of points on $H$ but not on the configurations $E_{n}$ or $D_{m}$. We obtain a new surface $X^{\prime \prime}$ with a negative configuration of curves consisting of $H^{\prime}$ (the strict transform of $H$ ) plus the curves coming from $E_{n}$ and $D_{m}$ (the negative definiteness is guaranteed by making $\left.H^{\prime 2}<<0\right)$. Now contract this negative configuration of curves, the singularity that is obtained by construction and using the previous corollary does not lie is a surface $X$ with $\operatorname{Kod}(X)=-\infty$.

\subsection{Symmetric powers.}

In this subsection, we show that any germ of an algebraic singularity $(X, s)$ can be realized in a projective variety $Y$ satisfying $Y_{\text {Sing }}=s$ and such that its smooth locus has abelian fundamental group. In particular, also $Y$ will have abelian fundamental group.

The construction will be based on the topological properties of symmetric powers of algebraic varieties. In fact $Y$ will be a generic complete intersection of $S^{2} X$.

For any CW-complex $X$ we can define a $m$-th symmetric power $S^{m} X$ as the quotient of the CW-complex $X^{m}=X \times \ldots \times X$ by the symmetric group of $m$-letters $S_{m}$. Hence $S^{m} X$ is also a CW-complex with a natural morphism $s_{m}: X^{m} \rightarrow S^{m} X$. For the sake of the readability of this paper, we recall some key topological properties of symmetric products with a short proof.

Lemma 3.2. Let $X$ be a $C W$-complex then the induced $C W$-complex $S^{m} X$ has the following properties.

(1) $\pi_{1}\left(S^{n} X\right)=H_{1}(X, \mathbb{Z}), n>1$ 
(2) $H^{i}\left(S^{l} X, \mathbb{R}\right)=H^{i}\left(S^{m} X, \mathbb{R}\right)$ for $m, l>i$

Proof: The cochains of $S^{m} X$ are symmetrizations of the cochains in the product of $m$ copies of $X$. Thus for $i<n$ symmetric cochains are generated by cochains in the product of $\leq i$ copies of $X$ multiplied by 0 -dimensional cochains. It implies that the $i$-skeletons of $S^{n} X, S^{m} X$ are isomorphic for $i<\min (m, n)$. Similarly the fundamental group of $S^{n} X$ is generated by the fundamental group of $X$.Any commutator relation corresponding to the cycle bounded by torus transforms in $S^{2} X$ by symmetric power which gives a disc.

If $X$ is an algebraic or projective variety then $S^{m} X$ is respectively an algebraic or projective variety. The variety $S^{m} X$ is singular unless $X$ is a nonsingular curve. Let us consider the case where $X$ is an algebraic variety of dimension $n$ with a finite collection of singular points $\Gamma=\left\{s_{1}, \ldots, s_{k}\right\}$. Denote $U=X_{\text {reg }}=X \backslash X_{\text {Sing }}$ and any of the $i$-diagonals of $U^{m}$ (the entries of a fixed set of $i$ places of $U^{m}$ are identical) by $\Delta_{i}$. We have the following Whitney stratification $\mathcal{S}$ of $S^{m} X$ :

(1) $\left(S^{m} X\right)_{\text {reg }}$.

(2) $P_{i}[m]=s_{m}\left(\Delta_{i+1}\right) \backslash \cup_{j=i+2}^{m} s_{m}\left(\Delta_{j}\right), 1 \leq i \leq m-1$.

(3) $\Sigma_{i}=S^{i}(\Gamma) \times\left(S^{m-i} U\right)_{\text {reg }} 1 \leq i \leq m$.

(4) $\Sigma P_{i j}=S^{i}(\Gamma) \times P_{j}[m-i], 1 \leq i \leq m$ and $1 \leq j \leq m-i$.

We denote the complement of the union of of all strata of codimension $\geq(i+1) n$ by $\left(S^{m} X\right)_{i}$. The $\left(S^{m} X\right)_{i}$ are Zariski open subsets of $S^{m} X$. For example, $\left(S^{m} X\right)_{0}=\left(S^{m} X\right)_{\text {reg }}$ and $\left.\left(S^{m} X\right)_{1}=S^{m} X\right)_{\text {reg }} \cup P_{1}[m] \cup \Sigma_{1}$. The following dimensional properties hold for the strata:

(1) $\operatorname{codim} P_{i}[m]=i n$, the singularities along $P_{i}[m]$ are simple quotient singularities.

(2) $\operatorname{codim} \Sigma_{i}=i n$.

(3) $\operatorname{codim} \Sigma P_{i j}=(i+j) n$.

We are now ready to state the main result of this section:

Theorem C. Let $\Gamma=\left\{\left(Y_{i}, y_{i}\right)\right\}_{i=1, \ldots, k}$ be any collection of germs of equidimensional isolated singularities. There exists a projective variety $X$ with abelian fundamental group whose collection of singular points coincide with $\Gamma$. Moreover if $Y$ is a projective variety with $Y_{\text {Sing }}=\Gamma$, then $X$ can be made such that $\pi_{1}(X \backslash \Gamma)=H_{1}(Y \backslash \Gamma, \mathbb{Z})$.

Proof. Let $Y$ be a projective variety whose collection of singular points coincide with $\Gamma$ (lemma 3.1). 
¿From lemma 3.2, it follows that the fundamental group of the symmetric product of any algebraic variety is abelian, and therefore we would like to take a generic complete intersection $Z$ in $S^{2} Y$ of the same dimension of $Y$ and that contains the same singularities of $Y$ in such a way that $\pi_{1}(Z)=$ $\pi_{1}\left(S^{2} Y\right)$. That would imply that $Z$ has an abelian fundamental group.

Lefschetz theorem on hyperplane sections states that if $W$ is an algebraic variety with $\operatorname{dim} W>2$ and $H \subset W$ is an hyperplane section such that $W \backslash H$ is smooth then $\pi_{1}(W)=\pi_{1}(H)$. For the variety $S^{2} Y$ that we are considering, Lefshetz theorem cannot be applied directly, because we want to study complete intersection subvarieties that are transverse to the singular locus of $S^{2} Y$.

Hence, let us consider $n$ generic hyperplane section $H_{1}, \ldots, H_{n}$ of $Y$, passing through the singular points of $Y$.

On the product $Y^{2}=Y \times Y$, let $p_{i}: Y^{2} \rightarrow Y$ with $i=1,2$, be the respective projections and let $H$ be the intersection of the divisors $p_{1}^{-1}\left(H_{j}\right) \cup$ $p_{2}^{-1}\left(H_{j}\right)$ with $j=1, \ldots n$.

Then $H$ is a complete intersection of very ample divisors on $Y^{2}$ that is invariant with respect of the natural action of the group $\mathbb{Z}_{2}$ on $Y^{2}$. We denote its quotient by

$$
Z=H / \mathbb{Z}_{2} \subseteq S^{2} Y
$$

Let $U$ be the smooth locus of $Y$, i.e. $U=Y \backslash \Gamma$, and let $H_{U}=H \cap(U \times U)$.

By applying Lefshetz theorem on $H_{U}$. it follows that $\pi_{1}\left(H_{U}\right)=\pi(U \times U)$ and since the action induced by $\mathbb{Z}_{2}$ on those groups is the same, we have that if $Z_{U}$ is the quotient of $H_{U}$ by $\mathbb{Z}_{2}$, then $Z_{U}$ has the same fundamental group of $S^{2} U$ and in particular this is abelian.

On the other hand $Z_{U}$ is also an open set of $Z$ such that its complementar is a union of a finite number of point and therefore the fundamental group $\pi_{1}(Z)$ is abelian since surjection $\pi_{1}\left(Z_{U}\right) \rightarrow \pi_{1}(Z)$ holds.

¿From the construction of $H$, it follows easily that the singularities of $Z$ are isolated and decompose into $Z_{\text {Sing }}=\left(Z \cap \Sigma_{1}\right) \cup\left(Z \cap P_{1}\right)$. The singularities in $Z \cap \Sigma_{1}$ are equivalent to the isolated singularities of $Y$ and the singularities in $Z \cap P_{1}$ are double points.

Let $X$ be the projective variety obtained from $Z$ by resolving the double points. Then $X$ has abelian fundamental group and its singular locus coincides with $\Gamma$, as desired.

Corollary 3.3. Let $Y$ be a projective variety with a given collection $\Gamma$ of isolated singular points such that $H_{1}(Y, \mathbb{Z})=0$. Then there exists a projective variety $X$ with $X_{\text {Sing }}=\Gamma$ which is simply connected. If additionally $Y$ is such that $H_{1}(Y \backslash \Gamma, \mathbb{Z})=0$ then $X$ can be made so that $X \backslash \Gamma$ is also simply connected. 


\section{Reducing abelian fundamental GRoup}

We are now ready to prove theorem A. Let $S$ be a given isolated singolarity. By the results in the previous section, we can suppose that there exists a variety $X$ such that $X_{\text {Sing }} \simeq S$, and, if $U=X \backslash X_{\text {Sing }}$, then $\pi_{1}(U)$ is abelian and the imbedding $U \hookrightarrow X$ defines a surjection $\pi_{1}(U) \rightarrow \pi_{1}(X)$.

Let us consider the infinite part of the fundamental group of $X$. If it is trivial, then the group is finite and hence there is a finite nonramified covering of $X$ which is simply connected.

Thus, we can suppose that the Albanese map $f: X \rightarrow A:=A l b(X)$ is not trivial. The torsion subgroup $\pi_{1}(X)_{\text {tors }}$ is a direct summand of $\pi_{1}(X)$, and therefore if $\pi_{1}(X)_{F}$ is the complementary subgroup, the induced morphism

$$
f_{*}: \pi_{1}(X)_{F} \rightarrow \pi_{1}(A)
$$

is an isomorphism.

For every $n>0$, we can consider the iteration map $f_{n}: S^{n} X \rightarrow A$, given by $f_{n}\left(x_{1} \ldots x_{n}\right)=\sum f\left(x_{i}\right)$. Since $f(X)$ generates $A$, there exists $n_{0}$ such that if $n \geq n_{0}$, then $f_{n}$ is surjective.

Moreover, we have

Lemma 4.1. There exists a positive integer $m>0$ such that the map $f_{m}$ : $S^{m} X \rightarrow A$ admits a topological section $s: A \rightarrow S^{m} X$.

Proof. Let $g=\operatorname{dim} A$, and let $\left[\gamma_{1}\right], \ldots,\left[\gamma_{2 g}\right]$ be generators of $\pi_{1}(A)$, given by considering the $i$-th component of $\Pi_{i=1}^{2 g} S^{1}$, for some homeomorphism $\Pi_{i=1}^{2 g} S^{1} \simeq A$.

Since the induced map $f_{*}: \pi_{1}(X) \rightarrow \pi_{1}(A)$ is surjective, for each $i=$ $1, \ldots, 2 g$, there exists an injective map $r_{i}: S^{1} \hookrightarrow X$, so that $f_{*}\left(r_{i}\right)=\gamma_{i}$. In particular, these maps induce an isomorphism $\pi_{1}(A) \rightarrow \pi_{1}(X)_{F}$.

The map $f_{2 g} \circ r: \Pi S_{i}^{1} \rightarrow A$, given by $f_{2 g} \circ r\left(z_{1}, \ldots, z_{2 g}\right)=\sum_{i=1}^{2 g} \gamma_{i}\left(z_{i}\right)$ is an homeomorphism and therefore the continuous map

$$
r=\Pi r_{i}: A \simeq \Pi S^{1} \rightarrow S^{2 g} X
$$

defines a topological section for $f_{2 g}: S^{2 g} X \rightarrow A$ (and in fact, more generally for $X^{2 g} \rightarrow A$ ).

Remark 4.2. The homotopy class of the section $s$ above, is defined by the homotopy class of the corresponding subgroup $\pi_{1}(X)_{F}$, generated by the elements $r_{i}: S^{1} \hookrightarrow X$.

Lemma 4.3. If $\operatorname{dim} X=n$, then for $N \geq i$, the natural imbedding $S^{i} X \hookrightarrow$ $S^{N} X$ is an homotopy equivalence up to dimension $i-1$.

Proof. By lemma 3.2, the statement is true for the fundamental group of $X$.

Moreover, it also holds for the homotopy groups $H^{i}\left(S^{i} X\right)$, since it is generated by products of elements in $H^{i_{s}}(X)$ with $i_{1}, \ldots, i_{k}$ so that their sum is equal to $i$ and hence all such product are represented on $S^{i} X$. 
In fact this is true on the level of complexes. Indeed the cells of dimension $i$ in $S^{N} X$ are obtained from the cells $i_{1}, \ldots, i_{k}$ with sum equal to $i$ in $X$. Thus each cell is the image of a product of at most $i$ simplices from $X$ and hence it comes from $S^{i} X$.

In particular the imbedding $S^{i} X \hookrightarrow S^{N} X$ is an homotopy equivalence up to dimension $i-1$.

By lemma 4.1, there exists $m>0$ and a topological section $s: A \rightarrow S^{m} X$. In particular, $f_{n}: S^{n} X \rightarrow A$ is surjective for any $n \geq m$.

Let $R_{x}^{n} \subseteq S^{n} X$ be the fiber of $f_{n}: S^{n} X \rightarrow A$ over a point $x \in A$. Moreover, let $\tilde{S}^{n} X \rightarrow S^{n} X$ be the abelian cover induced by the universal cover $\mathbb{C}^{g} \rightarrow A$, and let $\tilde{f}_{n}: \tilde{S}^{n} X \rightarrow \mathbb{C}^{g}$.

In particular, $\tilde{f}_{m}$ admits a topogical section $\tilde{s}: \mathbb{C}^{g} \rightarrow \tilde{S}^{m} X$, obtained as a cover of $s(A)$. Moreover the natural map $S^{n} X \times S^{k} X \rightarrow S^{n+k} X$, can be lifted to the map $\tilde{S}^{n} X \times \tilde{S}^{k} X \rightarrow \tilde{S}^{n+k} X$.

Lemma 4.4. The natural imbedding $i_{n}: R_{x}^{n} \hookrightarrow \tilde{S}^{n} X$ is an homotopy equivalence up to dimension $n-m-1$.

Proof. By lemma 4.3, the $k$-skeleton of $\tilde{S}^{m} X$ can be contracted to any subvariety

$$
\tilde{S}^{k+1} X \times c \subseteq \tilde{S}^{m} X
$$

where $c \in S^{m-k-1} X$ is any cycle.

Consider the map

$$
\phi_{k}: \tilde{S}^{k+1} X \rightarrow \tilde{S}^{k+1+m} X
$$

which maps $p \in \tilde{S}^{k+1}$ to $p \cdot \tilde{s}\left(-\tilde{f}_{k+1}(p)\right) \in \tilde{S}^{k+1+m}$.

Thus, we have

$$
\tilde{f}_{k+m+1}\left(\phi_{k}(p)\right)=\tilde{f}_{k+1}(p)+\tilde{f}_{m}\left(\tilde{s}\left(-\tilde{f}_{k+1}(p)\right)\right)=0 .
$$

Therefore $\phi_{k}$ maps $S^{k+1} X$ inside $\tilde{R}_{0}^{k+m+1}$.

Moreover it is homotopy equivalent to the standard imbedding. In fact the map

$$
(t, p) \mapsto p \cdot s\left(-t \tilde{f}_{k+1}(p)\right) \quad \text { for } 0 \leq t \leq 1
$$

defines the homotopy equivalence.

Thus $\tilde{S}^{k+1} X \subseteq \tilde{S}^{k+m+1} X$ is homotopy equivalent to its image in $R_{0}^{k+m+1}$, and therefore any nontrivial homotopy in $\tilde{S}^{k+m+1}$ is the same as in $R_{0}^{k+m+1}$, up to dimension $k$. This implies the lemma.

Remark 4.5. The same result and proof applies for any continuous map $a: S \rightarrow T$ from a topological space $S$ to a torus $T$, provided that the induced map $a^{\#}: \pi(S) \rightarrow \pi(T)$ is surjective and the map $\pi(S)^{a b} \rightarrow \pi(T)^{a b}$ is an isomorphism. 
Let $U \subseteq X$ be an open smooth subvariety of $X$, so that the natural map $\pi_{1}(U) \rightarrow \pi_{1}(X)$ is a surjection. Let $R U_{x}^{n} \subseteq R_{x}^{n}$ be the fiber for the induced map $S^{n} U \rightarrow A$. In particular, $R U_{x}^{n}$ is quasi-smooth, i.e. it has only quotient singularities and, by the same arguments used in lemma 4.4, the fibers $R U_{x}^{n}$ are homotopically equivalent up to dimension $n-m-1$.

Thus, if $n>m+1$ then the fundamental group of $R U_{x}^{n}$ is abelian and equal to the kernel of the map $\pi_{1}(U) \rightarrow \pi_{1}(A)$.

Fixed $x \in A$, let $M$ be the union of $R U_{x}^{n}$ with the intersection of $R_{x}^{n}$ and the image of the map $S^{n-1} U \times X_{\text {Sing }} \rightarrow S^{n} X$ then the resulting variety $M \subset R_{n}(X)$ has the following properties:

(1) $\pi_{1}(M)=\pi_{1}\left(R_{x}^{n}\right)$;

(2) $\operatorname{codim}\left(R_{x}^{n} \backslash M\right)>\operatorname{dim} X$;

(3) $\mathrm{M}$ is quasi-smooth outside a singular subset which is locally isomorphic to $\operatorname{Sing} X \times D$, where $D$ is a polydisk.

Thus if we take now a complete intersection of $M$ of dimension equal to the dimension of $X$ then the resulting variety $X^{\prime}$ will have isolated singularities which are the same as $X$ and $\pi_{1}\left(X^{\prime}\right)=\pi_{1}(M)$ and hence it is a finite abelian group. This finishes the construction and the proof.

\section{REFERENCES}

[A1] Artin M, On the solutions of analytic equations. Invent. Math. 5 (1968), 277-291.

[A2] Artin M, Algebraic approximation of structures over complete local rings . Publ. Math. I.H.E.S. 36 (1969), 23-58.

[BP] Bogomolov F.A., Pantev T., Weak Hironaka Theorem. Math. Res. Let. 3 (1996), 299-307.

[CG] Ciliberto C, Greco S, On normal surface singularities and a problem of Enriques. Communications Algebra 28 (2000 ) n.12, 5891-5913.

[EH] Epstein C, Henkin G, Stability of embeddings for pseudoconcave surfaces and their boundaries. Acta Math 185 (2000 ) n.2, 161-237.

[M] Mather J., Notes on Topological Stability, Mimeographed Notes, Harvard University (1970).

[Mo] Morrison D., The birational geometry of surfaces with rational double points. Math. Ann. 271 (1985), 415-438.

[H] Hartdt R., Topological Properties of subanalytic sets, Trans. Amer. Math. Soc. 211 (1975), 193-208.

[Le] Lempert L., Algebraic approximations in analytic geometry, Inv. Math., 121 (1995) n.2, 335-353.

[L] Levinson N., A polynomial canonical form for certain analytic functions of two variables at a critical point, Bull.Am. Math. Soc., 66 (1960), 366-368.

[S1] Sakai F., Weil divisors on normal surfaces. Duke Math. 51 (1984), 877-887.

[S1] Sakai F., The structure of normal surfaces. Duke Math. 52 (1985), 627-648.

[W] Whitney H., Local Properties of Analytic Varieties, Differential and Combinatorial Topology, Princeton Univ. Press, Princeton N.J. (1965), 205-244. 
Courant Institute of Mathematical Sciences, New York, NY 10012, USA, University of CAlifornia at SAnta Barbara, SANta Barbara, CA University of Miami, Department of mathematics, Coral Gables, FL 33124

E-mail address: bogomolo@cims.nyu.edu, cascini@cims.nyu.edu, bdeolive@math.miami.edu 